\title{
ARTIGO ORIGINAL Insatisfação com a imagem corporal em universitárias brasileiras
}

\author{
Body image dissatisfaction in female Brazilian university students \\ Marle dos Santos Alvarenga ,2, Sonia Tucunduva Philippi', Barbara H. Lourenç01, Priscila de Morais Sato³, \\ Fernanda Baeza Scagliusi ${ }^{3}$
}

\section{RESUMO}

A insatisfação com a imagem corporal é avaliada por meio da diferença entre a figura real e a idealizada e pode influenciar comportamentos alimentares. Objetivo: Avaliar a insatisfação corporal de universitárias do sexo feminino nas cinco regiões do país e possíveis associações e correlações com a idade, o estado nutricional, a renda e o grau de escolaridade do chefe da família. Métodos: 2.402 universitárias responderam à Escala de Silhuetas de Stunkard. As regiões foram comparadas por meio da análise de variância; correlações entre as variáveis foram analisadas pelos coeficientes de Pearson e Spearman. Resultados: 64,4\% gostariam de ser menores do que sua figura atual, e mesmo as estudantes eutróficas escolheram figuras

\section{Palavras-chave}

Insatisfação corporal, imagem corporal, estudantes universitárias.

\section{Keywords}

Body dissatisfaction, body image, college students. saudável e ideal menores. Na região Norte foram apontados os mais magros padrões ideais e de saúde e na região Centro-Oeste, os maiores. Conclusão: A ocorrência de insatisfação corporal foi bastante expressiva, com algumas diferenças regionais e sociodemográficas que devem ser consideradas.

\begin{abstract}
Body image dissatisfaction is evaluated according to the difference between the real figure and idealized figure, and could influence eating behaviors. Objective: To evaluate body dissatisfaction among female university students from the five regions of Brazil and possible associations and correlations with age, nutritional status, individual income and parental education. Methods: 2,402 students answered the Stunkard's Body Image Scale and regions were compared by variance analysis, and correlations among variables with Pearson's and Spearman's coefficients. Results: $64.4 \%$ desired a figure smaller than their actual one, and even the normal weight range students chose ideal and healthy smaller figures. In North region the ideal and healthy patterns were smaller and in the Central West bigger. Conclusion: Occurrence of body dissatisfaction was common among Brazilian university students and some regional and socio demographic differences must be considered.
\end{abstract}

1 Universidade de São Paulo (USP), Hospital das Clínicas, Instituto de Psiquiatria, Departamento de Psiquiatria, Ambulatório de Transtornos Alimentares (Ambulim).

2 Universidade de São Paulo (USP), Faculdade de Saúde Pública, Departamento de Nutrição.

3 Universidade Federal de São Paulo (Unifesp), Campus Baixada Santista, Departamento de Ciências da Saúde.

Endereço para correspondência: Marle dos Santos Alvarenga Departamento de Nutrição, FSP-USP

Av. Doutor Arnaldo, 715 - 01246-904 - São Paulo, SP

Telefone: (11) 3061-7803

E-mail: marlealvarenga@gmail.com 


\section{INTRODUÇÃO}

Imagem corporal é definida como a imagem que se tem na mente sobre o tamanho e a forma do próprio corpo, incluindo sentimentos em relação a essas características e às partes constituintes do corpo. Pode-se dividir a imagem corporal em dois componentes: o perceptivo - refere-se à imagem que é construída na mente - e o atitudinal - diz respeito aos sentimentos, pensamentos e ações em relação à imagem do corpo'.

Estudos sobre imagem corporal tentam entender quais fatores têm papel no desenvolvimento e na manutenção dos distúrbios da imagem corporal e dos transtornos alimentares (TA). Dentre esses se destacam os sociais, interpessoais e biológicos, como internalização dos ideais da mídia, respostas e comentários verbais negativos, maturação sexual precoce, abuso sexual, baixa autoestima e tendência à comparação muito grande entre a aparência das pessoas².

O ideal de corpo passou por mudança substancial no meio do século passado, com a propagação da atratividade representada como magreza para as mulheres e corpo musculoso para os homens. O sobrepeso passou a ser alvo de discriminação em vários meios e é estigmatizado como preguiça e falta de disciplina e motivação ${ }^{3}$. Embora os padrões de beleza mudem com o tempo, os atuais são onipresentes e estendem-se a toda a comunidade, além de serem inatingíveis para a maioria dos indivíduos. Muitas vezes, a pressão para atingir o suposto corpo ideal leva à piora da imagem corporal, aumento do comer desordenado e a tentativas mal-sucedidas de controle de peso. As influências socioculturais podem induzir ao desejo de um corpo magro e à insatisfação corporal, uma vez que não se consegue alcançar o ideal cultural ${ }^{3}$. Ironicamente, enquanto o corpo ideal continua a ser promovido pela mídia e pelo discurso cultural, a prevalência de sobrepeso e obesidade aumenta e coloca o indivíduo mais distante do ideal sociocultural ${ }^{4,5}$.

A insatisfação corporal é usada para avaliar a discrepância entre a imagem corporal real e a idealizada ${ }^{6}$. De modo geral, as mulheres apresentam maior insatisfação corporal que os homens, assim como maior prevalência de TA ${ }^{3}$. Aponta-se que a insatisfação corporal é associada com sintomas depressivos, estresse, baixa autoestima, maior restrição alimentar e evitação de atividade física ${ }^{7-9}$, indicando a importância de se avaliar esse parâmetro.

No Brasil, a magnitude da insatisfação corporal não tem sido profundamente estudada. Apenas estudos localizados, especialmente com adolescentes ou universitárias, avaliaram a satisfação com a imagem corporal a partir de questionários padronizados, como o Body Shape Questionnaire (BSQ) ${ }^{10-13}$; até onde se tem conhecimento, nenhum estudo trabalhou com o mesmo tipo de população para avaliar semelhanças ou diferenças entre as regiões do país.

Nesse contexto, o objetivo deste estudo foi avaliar a insatisfação corporal de universitárias do sexo feminino nas cinco diferentes regiões do país e possíveis associações ou correlações da imagem corporal com a idade, o estado nutricional, a renda individual e o grau de escolaridade do chefe da família.

\section{MÉTODOS}

Universitárias de 37 instituições de educação superior das cinco regiões do Brasil participaram do estudo, dentro de um projeto de pesquisa que avaliou insatisfação corporal e atitudes alimentares em estudantes da área da saúde (Alvarenga et al., submetido*).

Foram definidos os cursos de enfermagem e/ou psicologia por serem cursos eminentemente femininos; não havendo estes na instituição, foram indicados os de fonoaudiologia, fisioterapia, farmácia e/ou biomedicina. Foram convidadas estudantes do primeiro e segundo ano. Os critérios de inclusão foram: a) gênero feminino; b) idade maior ou igual a 18 anos e menor ou igual a 50 anos; c) assinatura do termo de consentimento. Os critérios de exclusão foram: a) ser nutricionista e/ou cursar graduação em nutrição; b) estar grávida. Estudantes de nutrição e nutricionistas foram excluídas, porque alguns estudos apontam que elas têm comportamento alimentar alterado 10,14,15; e pela proximidade com a área da nutrição e corpo, alunas de educação física não foram incluídas.

Os responsáveis locais receberam instruções específicas sobre a pesquisa e aplicação dos instrumentos, que deveriam ser autopreenchidos em sala de aula, juntamente com dados de caracterização como idade, peso e estatura (autorreferidos), renda individual e instrução do chefe da família.

A avaliação da satisfação corporal foi realizada por meio da Escala de Silhuetas de Stunkard ${ }^{16}$, que avalia:

- a percepção do tamanho e formas corporais: modo como o indivíduo enxerga e percebe seu tamanho ou sua figura mental do corpo atual;

- o ideal de tamanho e formas corporais: o que é considerado um "ideal" de beleza;

- a insatisfação com o tamanho e as formas corporais: diferença entre a percepção e o que considera ideal.

A versão para mulheres, adaptada no Brasil ${ }^{17}$, é uma escala simples, de fácil aplicação e avaliação. Para proceder à análise, cada figura recebe uma pontuação que varia de 0 , para a mais magra, a 9, para a mais obesa. A pessoa escoIhe uma figura que represente seu corpo atual (eu): quanto maior a pontuação, mais a pessoa se vê como obesa. Também escolhe uma figura para o corpo que desejaria ter (ideal): quanto menor a pontuação, mais se considera a magreza um ideal de beleza. O grau de insatisfação corporal é dado

\footnotetext{
*Alvarenga MS, Scagliusi FB, Philippi ST. Comparison of food attitudes among female college students from the five Brazilian regions. Cien Saude Colet. 2010. (submetido)
} 
pela diferença entre as figuras atual e ideal (número eu - número ideal). Os valores variam entre -8 e 8 (valores positivos expressam o desejo de ser mais magro e negativos, o desejo de ser mais gordo; "zero" corresponde à satisfação com o tamanho corporal atual).

$\mathrm{Na}$ versão em português, incluiu-se o questionamento de que figura o indivíduo considerava saudável para avaliar a diferença entre a figura eu e saudável (escore saudável), bem como a diferença entre as figuras saudável e ideal ${ }^{17}$.

Stunkard et al..$^{16}$ encontraram boa validade geral da Escala de Silhuetas, e estudo posterior ${ }^{18}$ atestou boa confiabilidade teste-reteste na seleção de figuras por mulheres $(r=0,71)$.

As análises estatísticas foram conduzidas com o software SPSS 12.0 (Statistical Package for the Social Science Inc., Chicago, Illinois USA). O nível de significância adotado foi de $p$ $<0,05$. Os resultados das variáveis de interesse eu, saudável, ideal, escore saudável (eu - saudável) e escore ideal (eu - ideal) são apresentados na forma de variáveis qualitativas, com frequências absolutas (n) e relativas (\%), e também como variáveis quantitativas (número da figura escolhida).

Para a comparação entre os números escolhidos para as variáveis de interesse, foi utilizada a análise de variância. As suposições de homocedasticidade foram verificadas e foi feito o ajuste por meio do teste de Brown-Forsythe, seguido de comparações múltiplas (dois a dois grupos), utilizando o teste de Dunnett, para identificar quais grupos apresentam diferenças entre si. A existência de correlação entre as variáveis de interesse entre si e com os demais parâmetros foi verificada com os coeficientes de correlação de Pearson e Spearman.

Uma análise de covariância foi realizada para verificar o possível impacto das covariáveis idade, estado nutricional, renda e escolaridade nas variáveis de interesse. As suposições de homocedasticidade foram verificadas e, quando houve diferença entre as regiões após o ajuste do modelo, foram feitas comparações múltiplas utilizando o teste de Tukey.

Comparações entre os valores escol hidos como eu versus ideal e eu versus saudável foram realizadas para saber quantas estudantes escolheram figuras ideais e saudáveis menores, iguais ou maiores do que sua figura eu. Esses valores foram contrapostos com a classificação do estado nutricional pelas categorias de índice de massa corpórea (IMC) da Organização Mundial de Saúde ${ }^{19} \mathrm{em}$ baixo peso (IMC $\left.<18,49 \mathrm{~kg} / \mathrm{m}^{2}\right)$, eutrofia (IMC entre 18,50 e $24,99 \mathrm{~kg} / \mathrm{m}^{2}$ ) e excesso de peso $\left(I M C>25 \mathrm{~kg} / \mathrm{m}^{2}\right)$. Para verificar se as categorias "saudável ou ideal < eu", "saudável ou ideal = eu", "saudável ou ideal > eu" eram diferentes entre regiões, faixas de renda, escolaridade e idade, foi utilizado o teste do qui-quadrado. Para os cruzamentos em que houve evidência de associação estatística, foi utilizada a análise de resíduo para a identificação de quais pares de categorias se diferenciam entre os grupos.

As participantes assinaram o Termo de Consentimento Livre e Esclarecido. A pesquisa foi aprovada pelo Comitê de Ética da Faculdade de Saúde Pública da Universidade de São Paulo.

\section{RESULTADOS}

O total de participantes na pesquisa geral (com objetivos além da satisfação corporal) foi de 2.489, e 2.402 alunas preencheram a Escala de Stunkard. Do total de participantes, 9,6\% eram da região Norte; $15,4 \%$, da região Nordeste; 8,0\%, da região Centro-Oeste; $35,6 \%$, da região Sudeste e 31,4\%, da região Sul; 58,7\% cursavam enfermagem; 15,1\%, psicologia; 12,3\%, farmácia; 9,4\%, fisioterapia; 1,8\%, biomedicina; 1,3\%, fonoaudiologia; e 1,4\% não informou o curso. Os dados descritivos da amostra estão na tabela 1.

Os resultados das respostas a Escala de Stunkard podem ser observados na tabela 2. Para a figura eu, os maiores valores estiveram no Sul, seguido pelo Sudeste e Norte. Para as figuras saudável e ideal, a escolha de figuras menores esteve no Norte e maiores no Centro-Oeste. A maior diferença entre as figuras eu e saudável (escore saudável) foi encontrada no Norte e a menor, no Centro-Oeste; o mesmo padrão de resposta foi encontrado em relação ao escore ideal, mas, nesse caso, sem diferença estatística.

Em relação ao número das silhuetas na Escala de Stunkard, as universitárias escolheram em média a figura 4 como representativa de seu corpo atual (eu), sendo as mais escolhidas a 4 (29,9\%), a 3 (21,3\%) e a 2 (19,8\%). Em média, as universitárias desejavam como ideal uma figura um número menor que aquele apontado como atual: as figuras ideais mais escolhidas foram a 3 (38,4\%) e a 2 (33,3\%), e a amplitude da diferença entre a figura eu e ideal variou de três figuras maiores a oito figuras menores. Quanto à figura escolhida como saudável, as mais frequentes foram a $3(42,1 \%)$, a $2(25,7 \%)$ e a $4(25,3 \%)$.

Do total nacional, 64,4\% das universitárias desejavam ser menores em algum grau $(21,8 \%$ escolheram como ideal a mesma figura que a eu e 13,9\%, uma figura maior). Além disso, 54,9\% escolheram como saudável uma figura menor que a eu $(29,9 \%$ escolheram como saudável a mesma que a figura eu e 15,2\%, uma figura maior).

A análise de correlação das variáveis de interesse entre si evidenciou correlação da variável eu com o escore saudável $(0,79)$ e escore ideal $(0,78)$ e do escore saudável com o escore ideal $(0,85)$. E a análise das variáveis com a idade mostrou fraca correlação $(-0,04$ a 0,18). Para com o IMC, encontraram-se correlações de 0,20 para ideal; 0,25 para saudável; 0,27 para idade; 0,60 para escore ideal; 0,63 para escore saudável e 0,72 para eu. A análise de correlação das variáveis de estudo com renda e escolaridade encontrou valores muito baixos $(-0,03$ a 0,06$)$.

A análise de covariância evidenciou que a idade, a escolaridade e o IMC influenciaram no modelo final da variável eu. No ajuste, a diferença permaneceu, com o Sul apresentando, em média, maior número do que as demais (Tabela 2). A idade e o IMC influenciaram a variável saudável e o modelo ajustado manteve as diferenças, com o Centro-Oeste apresentando, em média, número maior que as demais. A idade e o IMC influenciaram a variável ideal, mantendo-se 
as diferenças no modelo ajustado, com o Sul apresentando, em média, menor número do que o Norte e o Centro-Oeste (Tabela 2).

A escolaridade e o IMC influenciaram o escore saudável, mantendo-se a diferença no modelo ajustado, com diferença entre o Sul e as demais regiões - exceto a região Norte. A escolaridade e o IMC influenciaram o escore ideal, mas, quando ajustado, o modelo não mais apontou diferença entre as regiões (Tabela 2).

Em relação ao estado nutricional da amostra total ( $n=2.489$ ), encontrou-se que a maioria $(70,6 \%)$ era eutrófica; 9,2\% foram classificadas como abaixo do peso e 14,9\%, como acima do peso. Uma proporção delas (5,3\%) não informou o peso e/ou a altura - dados não demonstrados (Alvarenga et al., submetido).

A análise quanto à escolha da figura saudável em relação à figura eu, pelo estado nutricional, evidenciou que 38,7\% das eutróficas escolheram como saudável figuras menores do que a figura eu. Entre aquelas abaixo do peso, a maior parte escolheu figuras maiores e, entre as com excesso de peso, quase todas escolheram figuras menores (Tabela 3). Para a escolha da figura ideal em relação à figura eu, 47,8\% das eutróficas escolheram como ideal figuras menores do que eu. Entre aquelas abaixo do peso, a maior parte escolheu figuras maiores e, entre as com excesso de peso, a maioria escolheu figuras menores (Tabela 3).

A escolha saudável e ideal em relação à renda não mostrou diferença; e em relação ao grau de escolaridade, houve maior percentual de estudantes que escolheram a figura saudável > eu entre aquelas com chefe da família com escolaridade fundamental completo ou incompleto $(p=0,003)$. Na comparação entre ideal e eu, também houve maior percentual de estudantes que escolheram a figura ideal > eu entre aquelas com chefe com fundamental completo ou incompleto. Em contrapartida, entre aquelas com o chefe da família com curso superior completo ou incompleto, houve

Tabela 1. Idade, peso, altura, índice de massa corpórea da amostra de universitárias por região do país. Dados apresentados em média \pm desvio-padrão (mediana; mínimo - máximo)*

\begin{tabular}{|c|c|c|c|c|c|c|c|}
\hline Região & Norte (N) & Nordeste (NE) & Centro-0este (CO) & Sudeste (SE) & Sul(S) & Total nacional & $P$ \\
\hline Idade & $\begin{array}{l}26,1 \pm 7,1 \\
(24,0 ; 18-48)^{a} \\
n=231\end{array}$ & $\begin{array}{l}24,4 \pm 6,5 \\
(23 ; 18,0-50,0)^{b} \\
n=376\end{array}$ & $\begin{array}{l}21,9 \pm 5,4 \\
(20,0 ; 18,0-49,0)^{c} \\
n=193\end{array}$ & $\begin{array}{l}23,1 \pm 6,1 \\
(21,0 ; 18,0-50,0)^{d} \\
n=848\end{array}$ & $\begin{array}{l}23,0 \pm 5,3 \\
(21,0 ; 18,0-50,0)^{\mathrm{e}} \\
\mathrm{n}=776\end{array}$ & $\begin{array}{l}23,5 \pm 6,1 \\
(21,0 ; 18,0-50,0) \\
n=2424\end{array}$ & 0,001 \\
\hline Peso & $\begin{array}{l}58,5 \pm 10,5 \\
(57,0 ; 40,0-122,0) \\
n=227\end{array}$ & $\begin{array}{l}57,1 \pm 9,7 \\
(56,0 ; 37,0-118,0)^{f} \\
n=373\end{array}$ & $\begin{array}{l}57,2 \pm 9,0 \\
(56,0-38,0-98,0) \\
n=195\end{array}$ & $\begin{array}{l}59,2 \pm 10,2 \\
(58,0 ; 38,0-113,0)^{9} \\
n=856\end{array}$ & $\begin{array}{l}59,0 \pm 9,9 \\
(57,0 ; 39,0-120,0) \\
n=764\end{array}$ & $\begin{array}{l}58,6 \pm 10,0 \\
(57,0 ; 37,0-122,0) \\
n=2415\end{array}$ & 0,002 \\
\hline Altura & $\begin{array}{l}1,61 \pm 0,1 \\
(1,60 ; 1,48-1,82)^{h} \\
n=214\end{array}$ & $\begin{array}{l}1,62 \pm 0,1 \\
(1,61 ; 1,42-1,83)^{i} \\
n=368\end{array}$ & $\begin{array}{l}1,63 \pm 0,1 \\
(1,63 ; 1,49-1,81)^{j} \\
n=191\end{array}$ & $\begin{array}{l}1,64 \pm 0,1 \\
(1,63 ; 1,45-1,82)^{k} \\
n=852\end{array}$ & $\begin{array}{l}1,64 \pm 0,1 \\
(1,64 ; 1,46-1,84)^{\prime} \\
n=759\end{array}$ & $\begin{array}{l}1,63 \pm 0,1 \\
(1,63 ; 1,42-1,84) \\
n=2384\end{array}$ & 0,000 \\
\hline IMC & $\begin{array}{l}22,7 \pm 3,5 \\
(22,1 ; 16,4-46,0)^{m} \\
n=211\end{array}$ & $\begin{array}{l}21,9 \pm 3,4 \\
(21,4 ; 14,2-41,8) \\
n=364\end{array}$ & $\begin{array}{l}21,5 \pm 3,1 \\
(21,1 ; 16,6-33,3)^{n} \\
n=191\end{array}$ & $\begin{array}{l}22,2 \pm 3,7 \\
(21,4 ; 16,1-49,6) \\
n=841\end{array}$ & $\begin{array}{l}21,9 \pm 3,3 \\
(21,2 ; 15,2-41,5) \\
n=750\end{array}$ & $\begin{array}{l}22,0 \pm 3,5 \\
(21,4 ; 14,2-49,6) \\
n=2357\end{array}$ & 0,005 \\
\hline
\end{tabular}

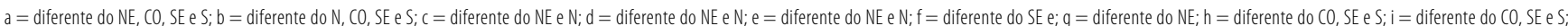
$\mathrm{j}=$ diferente do $N E$ e $N ; k$ = diferente do $N E$ e $N ; I=$ diferente do NE e $N ; m=$ diferente do $C 0 ; n=$ diferente do $N$.

* Nem todas as alunas informaram idade, peso e altura; assim, o número total válido de respostas está indicado por região.

Tabela 2. Dados descritivos das figuras escolhidas como Eu, Saudável e Ideal e Escore Saudável (figura eu - figura saudável) e Escore ldeal (figura eu - figura ideal) por região do país. Dados apresentados em média \pm desvio-padrão (mediana)*

\begin{tabular}{|c|c|c|c|c|c|c|c|}
\hline Região & Norte (N) & Nordeste (NE) & Centro-Oeste (CO) & Sudeste (SE) & Sul(S) & Total nacional & $P$ \\
\hline Figura eu & $\begin{array}{l}3,66 \pm 1,34 \\
(4)^{a} n=229\end{array}$ & $\begin{array}{l}3,55 \pm 1,36 \\
(4)^{b} \mathrm{n}=370\end{array}$ & $\begin{array}{l}3,53 \pm 1,38 \\
\text { (4) } n=182\end{array}$ & $\begin{array}{l}3,67 \pm 1,45 \\
(4) n=864\end{array}$ & $\begin{array}{l}3,77 \pm 1,40 \\
(4)^{c} n=757\end{array}$ & $\begin{array}{l}3,67 \pm 1,41 \\
(4) n=2402\end{array}$ & $\begin{array}{l}\text { NxS }=0,001 \text { NExS }<0,001 \\
\text { SExS }<0,001\end{array}$ \\
\hline Figura saudável & $\begin{array}{l}2,86 \pm 0,97 \\
(3)^{d} n=213\end{array}$ & $\begin{array}{l}2,96 \pm 0,88 \\
(3)^{\mathrm{e}} \mathrm{n}=311\end{array}$ & $\begin{array}{l}3,22 \pm 0,88 \\
(3)^{f} n=147\end{array}$ & $\begin{array}{l}3,02 \pm 0,89 \\
(3)^{9} n=784\end{array}$ & $\begin{array}{l}3,06 \pm 0,87 \\
(3)^{h} n=680\end{array}$ & $\begin{array}{l}3,02 \pm 0,89 \\
(3) n=2135\end{array}$ & $\begin{array}{l}\mathrm{NxCO}=0,001 \\
\mathrm{~N} x \mathrm{~S}=0,032 \\
\mathrm{NExCO}=0,002 \\
\mathrm{COxSE}=0,010\end{array}$ \\
\hline Figura ideal & $\begin{array}{l}2,56 \pm 0,97 \\
(3)^{i} \mathrm{n}=218\end{array}$ & $\begin{array}{l}2,74 \pm 0,89 \\
\text { (3) } n=315\end{array}$ & $\begin{array}{l}2,81 \pm 0,90 \\
(3)^{j} \mathrm{n}=155\end{array}$ & $\begin{array}{l}2,74 \pm 0,93 \\
(3) n=778\end{array}$ & $\begin{array}{l}2,80 \pm 0,91 \\
(3)^{k} \mathrm{n}=699\end{array}$ & $\begin{array}{l}2,74 \pm 0,92 \\
(3) n=2165\end{array}$ & $\begin{array}{l}\mathrm{N} x \mathrm{CO}=0,035 \\
\mathrm{~N} \times \mathrm{S}=0,008\end{array}$ \\
\hline Escore saudável & $\begin{array}{l}0,79 \pm 1,25 \\
(1) n=211\end{array}$ & $\begin{array}{l}0,57 \pm 1,35 \\
(1)^{\prime} n=310\end{array}$ & $\begin{array}{l}0,38 \pm 1,32 \\
(0)^{m} n=147\end{array}$ & $\begin{array}{l}0,68 \pm 1,29 \\
(1)^{\mathrm{n}} \mathrm{n}=779\end{array}$ & $\begin{array}{l}0,77 \pm 1,18 \\
(1) n=678\end{array}$ & $\begin{array}{l}0,69 \pm 1,27 \\
(1)^{\circ} n=2125\end{array}$ & $\begin{array}{l}\text { NEXS }=0,033 \\
C 0 \times S=0,022 \\
\text { SExS }=0,006\end{array}$ \\
\hline Escore ideal & $\begin{array}{l}1,08 \pm 1,25 \\
\text { (1) } n=217\end{array}$ & $\begin{array}{l}0,80 \pm 1,48 \\
\text { (1) } n=314\end{array}$ & $\begin{array}{l}0,75 \pm 1,45 \\
\text { (1) } n=155\end{array}$ & $\begin{array}{l}0,94 \pm 1,37 \\
(1) n=772\end{array}$ & $\begin{array}{l}1,02 \pm 1,33 \\
(1) n=695\end{array}$ & $\begin{array}{l}0,95 \pm 1,37 \\
\text { (1) } n=2153\end{array}$ & 0,050 \\
\hline
\end{tabular}

$\mathrm{a}=$ diferente do $\mathrm{S} ; \mathrm{b}=$ diferente do $\mathrm{S} ; \mathrm{c}=$ diferente do $\mathrm{N}$ e NE; $\mathrm{d}=$ diferente do $\mathrm{CO}$ e $\mathrm{S} ; \mathrm{e}=$ diferente do $\mathrm{CO} ; \mathrm{f}=$ diferente do $\mathrm{N}, \mathrm{NE}$ e $\mathrm{SE} ; \mathrm{g}=$ diferente do $\mathrm{CO} ; \mathrm{h}=$ diferente do $\mathrm{N} ; \mathrm{i}=$ diferente $\mathrm{CO}$ e $\mathrm{S} ; \mathrm{j}=$ diferente do $\mathrm{N} ; \mathrm{k}=$ diferente do $\mathrm{N}$; $I=$ diferente do $S ; m=$ diferente do $S ; n=$ diferente do $S ; 0=$ diferente do NE, CO e SE.

* Nem todas as alunas preencheram as três opções solicitadas de resposta: eu, ideal e saudável, assim, o número total válido de respostas está indicado por região. 
Tabela 3. Dados de escolha da figura Saudável e da figura Ideal em relação à figura Eu de acordo com a Classificação do Estado Nutricional - de acordo com o índice de massa corpórea - do total da amostra de universitárias brasileiras

\begin{tabular}{|c|c|c|c|c|c|c|}
\hline & & & & Iice de massa corpórea - IMC ( $(\mathrm{kg} / \mathrm{h}$ & & Tet \\
\hline & & & Abaixo do peso $($ (IMC $<18,49)$ & Peso normal (IMC 18,5 a 24,99$)$ & Acima do peso (IMC $>25,00)$ & Total \\
\hline & caud́́n < ou & $\mathrm{N}$ & 20 & 781 & 306 & 1107 \\
\hline & $\underline{\text { Saluavel }}<\underline{\text { eu }}$ & $\%$ & $1,0 \%$ & $38,7 \%$ & $15,2 \%$ & $54,9 \%$ \\
\hline & & $\mathrm{N}$ & 54 & 537 & 10 & 601 \\
\hline & & $\%$ & $2,7 \%$ & $26,6 \%$ & $0,5 \%$ & $29,8 \%$ \\
\hline & & $\mathrm{N}$ & 124 & 186 & 0 & 310 \\
\hline & Saquavel tw & $\%$ & $6,1 \%$ & $9,2 \%$ & $0,0 \%$ & $15,4 \%$ \\
\hline Totot & & $\mathrm{N}$ & 198 & 1504 & 316 & 2018 \\
\hline & & $\%$ & $9,8 \%$ & $74,5 \%$ & $15,7 \%$ & $100,0 \%$ \\
\hline & & $\mathrm{N}$ & 26 & 978 & 310 & 1314 \\
\hline & $\underline{\underline{t u d a}}<\underline{\mathrm{u}}$ & $\%$ & $1,3 \%$ & $47,8 \%$ & $15,1 \%$ & $64,2 \%$ \\
\hline Comn & & $\mathrm{N}$ & 43 & 391 & 12 & 446 \\
\hline 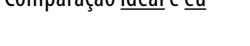 & $\underline{1 \text { netal }}-\underline{\underline{u}}$ & $\%$ & $2,1 \%$ & $19,1 \%$ & $0,6 \%$ & $21,8 \%$ \\
\hline & & $\mathrm{N}$ & 131 & 156 & 0 & 287 \\
\hline & - & $\%$ & $6,4 \%$ & $7,6 \%$ & $0,0 \%$ & $14,0 \%$ \\
\hline Tatat & & $\mathrm{N}$ & 200 & 1525 & 322 & 2047 \\
\hline & & $\%$ & $9,8 \%$ & $74,5 \%$ & $15,7 \%$ & $100,0 \%$ \\
\hline
\end{tabular}

maior proporção de estudantes que escolheram como ideal figuras menores do que eu $(p=0,04)$.

Houve maior percentual de estudantes que escolheram como saudável e ideal figuras menores do que eu entre aquelas com idade acima de 25 anos. Paralelamente, houve maior percentual de estudantes com idade até 19 anos que escolheram como saudável e ideal figuras maiores ou iguais a eu $(p<0,001)$.

Quando esses valores foram comparados em relação à região, houve maior percentual de estudantes que escolheram a figura saudável > eu no Centro-Oeste e no Nordeste $(p=0,001)$. Na comparação entre ideal e eu, houve maior percentual de estudantes que escolheram figura ideal > eu também no Centro-Oeste e no Nordeste $(p=0,005)$.

\section{DISCUSSÃO}

Os resultados apontam que a maioria das universitárias $(64,4 \%)$ desejava ser menor do que é, atestando grande magnitude de insatisfação corporal nesse grupo. Deve-se considerar na interpretação desse resultado a ampla dimensão do grupo analisado, incluindo estudantes de todas as regiões do Brasil para avaliar esse parâmetro. Até onde se conhece, este é o único trabalho nacional que comparou um mesmo grupo etário em relação à imagem corporal em diferentes regiões do país.

A metodologia de avaliação utilizada nesta pesquisa é diferente da de outros trabalhos nacionais avaliando imagem corporal, portanto é preciso comparar os resultados com cautela. Entre os estudos nacionais que avaliaram imagem corporal em universitárias, Souza et al. ${ }^{20}$ utilizaram a metodologia da autocrítica corpórea para avaliar estudantes de medicina no Ceará, encontrando 28,1\% com distorção da imagem corporal. Kakeshita e Almeida ${ }^{21}$, avaliando universitários em São Paulo com uma escala de figuras desenvolvida para o trabalho em questão, encontraram que $87 \%$ das mulheres eutróficas ou com sobrepeso superestimaram seu tamanho corporal, indicando distorção na autopercepção da imagem.

Avaliando satisfação com a imagem corporal utilizando o BSQ, Moreira et al..$^{13}$ encontraram que 50\% das estudantes na Bahia apresentavam algum grau de insatisfação corporal. Bosi et al. ${ }^{10}$ encontraram $40,4 \%$ de insatisfação em estudantes de nutrição e $17,1 \%$ e 18,6\% com insatisfação moderada ou grave em estudantes de educação física e psicologia, respectivamente ${ }^{11,12}$.

Validando a Escala de Stunkard no Brasil, foram escolhidas pelo grupo de universitárias de nutrição em média a figura $3,5 \pm 1,3$ (mediana 4) como eu e a figura 2,8 $\pm 1,0$ (mediana 3) como ideal; o escore eu-ideal foi de $0,7 \pm 1,3$ (mediana 1 ) - valor menor do que o encontrado em média neste estudo $(0,95 \pm 1,37)$, mas com igual mediana ${ }^{17}$.

No presente trabalho, em média, as estudantes escoIheram como saudável figuras maiores do que as que escoIheram como ideal. Estudantes de baixo peso escolheram figuras ideal e saudável maiores do que aquelas que apontam como atual, ao passo que aquelas acima do peso escoIheram figuras ideal e saudável menores do que as atuais. 
No entanto, grande número de estudantes eutróficas escoIheu como ideal e saudável números menores do que a figura atual, evidenciando que a insatisfação corporal é grande mesmo naquelas de peso normal. Deve-se considerar que este estudo tem a limitação de não ter trabalhado com um cálculo de amostragem para o desfecho insatisfação corporal, e sim com atitudes alimentares desordenadas (Alvarenga et al., submetido). No entanto, acredita-se que, pelo número expressivo de participantes e pela inexistência de trabalhos amplos sobre essa temática no país, pode-se obter um perfil da insatisfação corporal de universitárias de diferentes regiões brasileiras a partir desses dados.

Os maiores valores para a figura eu estiveram no Sul, mesmo não sendo essa a região com maior IMC e tendose encontrado alta correlação $(0,72)$ entre a figura eu e o IMC. Dessa forma, pode-se inferir que as estudantes do Sul parecem se ver maiores que as demais. Já os menores valores apontados como ideal e saudável estiveram no Norte, enquanto os maiores valores foram provenientes do CentroOeste (mesmo a diferença não sendo igual em relação a todas as demais regiões). É possível afirmar, portanto, que as estudantes do Norte têm parâmetros de ideal de beleza e de saúde mais magros e que as do Centro-Oeste possuem parâmetros maiores. A maior diferença entre o atual e o saudável foi encontrada também no Norte do país, mas para a diferença entre o atual e ideal não houve distinção entre as regiões - a análise de variância demonstrou que as diferenças eram devidas à escolaridade e ao IMC.

Essa diferença encontrada na região Norte não pode ser explicada pela idade, renda, IMC ou escolaridade do chefe da família. Esse mesmo padrão de resposta - pior resultado no Norte - foi encontrado na avaliação de atitudes alimentares desse grupo (Alvarenga et al., submetido). Avaliações em relação ao comer desordenado e influência da mídia não são feitas comumente com populações do Norte, ou mesmo comparações entre regiões. Sugere-se a ampliação dos estudos sobre comportamento alimentar e relação com o corpo nessa região, que ainda é desconhecida em relação a uma série de fatores.

Não houve correlação forte entre as figuras escolhidas nas silhuetas com idade, renda ou escolaridade do chefe da família. Correlações mais significativas foram encontradas apenas em relação ao IMC, especialmente para a escolha das figuras eu e para os escores saudável e ideal. Assim, parece que as estudantes têm uma percepção razoável de seu tamanho atual e que este pode influenciar na diferença entre a figura que escolhem como saudável e ideal.

A correlação encontrada entre a figura eu e as figuras saudável e ideal foi forte e positiva, e ainda mais a correlação do escore saudável com o escore ideal. Esse achado evidencia que, dependendo da percepção de seu tamanho atual, as estudantes fazem determinadas escolhas em relação ao que consideram saudável e ideal. Esse resultado é reforça- do quando, na análise dessas figuras por faixas de IMC, se observa que estudantes de baixo peso escolheram figuras maiores como sendo saudável e ideal, enquanto aquelas com excesso de peso escolheram figuras menores, o que seria "adequado" e "esperado" para esses grupos. Já a correlação entre os dois escores mostra que, embora o número médio escolhido para ideal seja um pouco menor do que o saudável, as escolhas tendem a ser muito similares para esses parâmetros - nomeia-se de saudável aquilo que escolhe como ideal e vice-versa.

Além da magnitude da insatisfação corporal, chama atenção o fato de que mesmo as eutróficas desejam ser menores e, além de desejarem um corpo mais magro, a escolha de uma figura saudável menor faz discutir o conceito de saúde e boa forma vigente, muito associado a uma magreza não saudável de acordo com os parâmetros médicos. Essa situação atesta o descontentamento normativo apontado entre as mulheres da sociedade contemporânea - todas acreditam que ficariam mais bonitas e atraentes se perdessem algum peso ${ }^{22}$. Sabese que não apenas a mulher obesa, e sim a mulher ocidental contemporânea, como um todo, está insatisfeita com sua imagem corporal. Segundo Ortega et al.23, 31,6\% das estudantes espanholas com IMC $<20,1 \mathrm{~kg} / \mathrm{m}^{2}$ queriam perder peso. Analisando mulheres (20-30 anos) ingressantes em uma academia de ginástica em Curitiba, Filardo e Leite ${ }^{24}$ observaram que, embora 55\% desejassem a redução de peso, somente $17 \%$ delas realmente necessitavam. A insatisfação com o próprio corpo parece estar relacionada às exigências sociais e culturais de aparência e magreza; as pessoas parecem tender, de forma estereotipada, a avaliar a imagem corporal em função das normas sociais e culturais de peso idea|25-27.

A análise em relação às figuras e ao estado nutricional evidenciou ainda que as estudantes com chefes de família com menor escolaridade e mais jovens escolheram figuras saudáveis e ideais maiores que as atuais, enquanto estudantes com chefes de família de escolaridade superior e mais velhas pareceram ter parâmetros mais magros para ideal e saudável. A escolaridade do chefe de família pode ser um indicativo da condição socioeconômica e, nesse sentido, o presente estudo confirmaria a ideia geral de que o ideal de beleza se aplica mais incisivamente a indivíduos de estratos econômicos mais privilegiados. Contudo, estudo de Snapp ${ }^{28}$ demonstrou que a internalização dos padrões socioculturais de beleza é comprovada em diferentes etnias e classes sociais, incluindo adolescentes entre 14 e 18 anos provenientes de estratos menos favorecidos.

Essa evidência também auxilia a discussão da diferença nos níveis de insatisfação corporal em relação à idade. Enquanto se pode considerar consenso na literatura que o padrão de beleza dissemina-se mesmo em faixas etárias muito jovens ${ }^{29}$, é preciso lembrar que esse padrão de beleza é estrito e pouco democrático, favorecendo, além de formas corporais mais magras, mulheres mais jovens. Sendo assim, é 
plausível inferir que uma maior carga de exigências estéticas possa incidir sobre mulheres mais velhas, justificando a escoIha de figuras mais magras.

Essa mesma análise em relação ao estado nutricional por região do país evidenciou que o Nordeste e o Centro-Oeste apresentaram escolhas menos magras. Não se pode explicar essa diferença facilmente, mas, novamente, ao se fazer uma comparação com os dados de atitudes alimentares, o estudo com esse grupo encontrou que a região Centro-Oeste apresentou os melhores escores para esse atributo (Alvarenga et al., submetido), reforçando a possibilidade de associação entre atitudes alimentares e insatisfação corporal. Como os parâmetros analisados não foram capazes de desvendar por que estudantes de algumas regiões teriam ideais menos magros dos que outras, faz-se necessário o estudo de uma gama mais ampla de variáveis atreladas ao desenvolvimento da insatisfação corporal, como fatores relacionados à disseminação e à internalização de padrões socioculturais de beleza (influência da mídia, pressão exercida por familiares e pares).

Os distúrbios da imagem corporal são fatores bem conhecidos para níveis clínicos ou subclínicos de TA². Sabe-se, ainda, que a insatisfação corporal influencia fortemente a prática de dietas e outras estratégias restritivas ${ }^{2,30}$. Estes resultados chamam a atenção, portanto, para a importância de estratégias de prevenção de TA em universitárias do país. A identificação de fatores de risco para insatisfação corporal e TA é essencial para o desenvolvimento e a implantação de programas efetivos de tratamento e prevenção ${ }^{31}$; o conhecimento apropriado da realidade da insatisfação corporal e também de atitudes alimentares inadequadas deve direcionar estratégias de educação nutricional e de promoção à saúde para mulheres jovens no contexto nacional.

\section{CONCLUSÃO}

A amostra de universitárias brasileiras apresentou relevante insatisfação corporal, e algumas interessantes diferenças regionais foram encontradas: estudantes do Norte tiveram os ideais mais magros e as do Centro-Oeste, os maiores ideais para corpo saudável e desejável. As universitárias parecem ter uma percepção razoável de seu tamanho atual e isso pareceu influenciar a diferença entre a figura que escolhem como saudável e ideal. O grau de insatisfação presente mesmo entre as eutróficas deve ser considerado em programas de promoção à saúde e à educação sobre comportamentos e métodos adequados para manutenção do peso corporal.

\section{AGRADECIMENTOS}

Os autores agradecem à Fundação de Amparo à Pesquisa do Estado de São Paulo (Fapesp) pelo auxílio à realização des- ta pesquisa (processo 06/56850-9). Agradecem também a expressiva colaboração dos coordenadores do curso de nutrição e seus parceiros na coleta de dados nas instituições participantes: Centro de Ensino Unificado de Teresina, Centro Universitário de Barra Mansa, Centro Universitário de Brasília, Centro Universitário de Vila Velha, Centro Universitário de Volta Redonda, Centro Universitário do Sul de Minas, Centro Universitário Feevale, Centro Universitário La Salle, Centro Universitário Nilton Lins, Faculdade Itabirana de Saúde, Faculdade Natalense para o Desenvolvimento do Rio Grande do Norte, Faculdade São Lucas, Faculdade Vale do Ipojuca, Pontifícia Universidade Católica do Paraná, Unidade de Ensino Superior do Sul do Maranhão, Universidade Católica Dom Bosco, Universidade Cruzeiro do Sul, Universidade de Brasília, Universidade de Caxias do Sul, Universidade de Cuiabá, Universidade do Sul de Santa Catarina, Universidade do Vale do Itajaí, Universidade do Vale do Rio dos Sinos, Universidade Federal de Alfenas, Universidade Federal de Pelotas, Universidade Federal de Santa Catarina, Universidade Federal do Mato Grosso, Universidade Federal do Paraná, Universidade Federal do Piauí, Universidade Federal do Rio Grande do Sul, Universidade Metodista de Piracicaba, Universidade Metodista de São Paulo, Universidade Municipal de São Caetano do Sul, Universidade Paulista Campus Manaus/São Paulo - Capital/Sorocaba.

\section{REFERÊNCIAS}

1. Slade PD. What is body image? Behav Resear Ther. 1994;32(5):497-502.

2. Van Den Berg P, Thompson JK, Obremski-Brandon K, Coovert M. The Tripartite influence model of body image and eating disturbance - a covariance structure modeling investigation testing the mediational role of appearance comparison. J Psychosom Res. 2002;53(5):1007-20.

3. Neighbors $L A$, Sobal J. Prevalence and magnitude of body weight and shape dissatisfaction among university students. Eat Behav. 2007;8(4):429-39.

4. Ogden CL, Carroll MD, Curtin LR, McDowell MA, Tabak CJ, Flegal KM. Prevalence of overweight and obesity in the United States, 1999-2004. JAMA. 2006;295(13):1549-55.

5. Polivy J. Psychological consequences of food restriction. J Am Diet Assoc. 1996;96(6):589-92.

6. Thompson JK, Van Den Berg P. Measuring body image attitudes among adolescents and adults. In: Cash TF, Pruzinsky T, editors. Body image: a handbook of theory, research and clinical practice. The Guilford Press. New York; 2002, p. 142-54.

7. Anton SD, Perri MG, Riley JR. Discrepancy between actual and ideal images: impact on eating and exercise behaviors. Eat Behav. 2000;1(2):153-60.

8. Johnson F, Wardle J. Dietary restraint, body dissatisfaction, and psychological distress: a prospective analysis. J Abnorm Psychol. 2005;114(1):119-25.

9. Markey CN, Markey PM. Relations between body image and dieting behaviors: an examination of gender differences. Sex Roles. 2005;53(7/8):519-30.

10. Bosi MLM, Luiz RR, Morgado CMC, Costa MLS, Carvalho RJ. Autopercepção da imagem corporal entre estudantes de nutrição no Rio de Janeiro. J Bras Psiquiatr. 2006;55(1):34-40.

11. Bosi MLM, Luiz RR, Uchimura KY, Oliveira FP. Comportamento alimentar e imagem corporal entre estudantes de educação física. J Bras Psiquiatr. 2008;57(1):28-33.

12. Bosi MLM, Uchimura KY, Luiz RR. Eating behavior and body image among psychology students. J Bras Psiquiatr. 2009;58(3):150-5.

13. Moreira $L A C$, Azevedo $A B G$, Queiroz D, Moura L, Santo DE, Cruz R, et al. Body image in a sample of undergraduate medical students from Salvador, Bahia, Brazil. J Bras Psiquiatr. 2005;54(4):295-7. 
14. Fiates GMR, Salles RK. Fatores de risco para o desenvolvimento de distúrbios alimentares: um estudo em universitárias. Rev Nutr. 2001;14(Supl):3-6.

15. Fredenberg JP, Berglund PT, Dieken HA. Incidence of eating disorders among selected female university students. J Am Diet Assoc. 1996;96(1):64-5.

16. Stunkard A, Sorensen T, Schlusinger F. Use of Danish adoption register for the study of obesity and thinness. In: Kety S, Rowland LP, Sidman RL, Matthysse SW, editors. The genetics of neurological and psychiatric disorders. New York: Raven; 1983. p. 115-20.

17. Scagliusi FB, Alvarenga M, Polacow V0, Cordás TA, Queiróz GKO, Coelho D, et al. Concurrent and discriminant validity of the Stunkard's Figure Rating Scale adapted into Portuguese. Appetite. 2006;47(1):77-82.

18. Thompson JK, Altabe M. Psychometric qualities of the figure rating scale. Int J Eat Disord. 1991;10(5):615-9.

19. Organização Mundial da Saúde (OMS). Global database on Body Mass Index. WHO, Geneva, 2006. Disponível em: http://apps.who.int/bmi/index.jsp?introPage=intro_3.html. Acessado em: 20 out. 2009.

20. Souza FGM, Martins MCR, Monteiro FCC, Neto GCM, Ribeiro IB. Anorexia e bulimia nervosa em alunas da Faculdade de Medicina da Universidade Federal do Ceará. Rev Psiquiatr Clin. 2002;24(4):172-80.

21. Kakeshita IS, Almeida SS. Relação entre índice de massa corporal e a percepção da autoimagem em universitários. Rev Saude Publica. 2006;40(3):497-504.

22. Rodin J, Silberstein L, Striegel-Moore RH. Women and weight: a normative discontent. In: Sonderegger TB, Anastasi A, eds. Psychology and gender. Lincoln, NE: University of Nebraska Press; 1985, p. 245-307.
23. Ortega RM, Requejo AM, Quintas E, Sanchez-Quiles B, Lopez-Sobaler AM, Andres P. Estimated energy balance in female university students: differences with respect to body mass index and concern about body weight. Int J Obes Relat Metab Disord. 1996;20(12):1127-9.

24. Filardo RD, Leite N. Perfil dos indivíduos que iniciam programas de exercícios em academias, quanto à composição corporal e aos objetivos em relação à faixa etária e sexo. Rev Bras Med Esporte. 2001;7(2):57-61.

25. Ogden J, Evans C. The problem with weighing: effects on mood, self-esteem and body image. Int J Obes Relat Metab Disord. 1996;20(3):272-7.

26. Foster $G D$, Wadden $T A$, Vogt RA. Body image in obese before, during, and after weight loss treatment. Health Psychol. 1997;16(3):226-9.

27. Leonhard ML, Barry NJ. Body image and obesity: effects of gender and weight on perceptual measures of body image. Addict Behav. 1998;23(1):31-4.

28. Snapp $S$. Internalization of the thin ideal among low-income ethic minority adolescent girls. Body Image. 2009;6(4):311-4.

29. Dittmar H, Halliwell E, Ive S. Does Barbie make girls want to be thin? The effect of experimental exposure to images of dolls on the body image of 5- to 8-year-old girls. Dev Psychol. 2006;42(2):283-92.

30. Thompson JK, Smolak L. Body Image, eating disorders, and obesity in youth: assessment, prevention, and treatment. Developmental Psychology: American Psychological Association; 2001.

31. Thompson JK, Van Den Berg P, Roehrig M, Guarda AS, Heinber LJ. The sociocultural attitudes towards appearance scale-3 (SATAQ-3): development and validation. Int J Eat Disord. 2004;35(3):293-304. 